\title{
MBLDP-R: A Multiple Biomolecules Based Rapid Life Detection Protocol Embedded in a Rover Scientific Subsystem for Soil Sample Analysis
}

\section{Akib Zaman}

Military Institute of Science and Technology

Fardeen Ashraf

Military Institute of Science and Technology

Haseena Khan

University of Dhaka

Faria Noshin

University of Dhaka

Oiliullah Samir

Military Institute of Science and Technology

Asif Mahmud Rayhan

Military Institute of Science and Technology

Sadia Nur Nazifa

Military Institute of Science and Technology

Hafsah Mahzabin Chowdhury

Military Institute of Science and Technology

Mahbubur Rahman ( $\nabla$ mahbub@cse.mist.ac.bd)

Military Institute of Science and Technology

\section{Research Article}

Keywords: Life detection, Multiple biomolecule integration, Qualitative test-scoring, Life-detection rover subsystem, Computer-aided analysis

Posted Date: January 20th, 2022

DOI: https://doi.org/10.21203/rs.3.rs-1263964/v1

License: (c) (1) This work is licensed under a Creative Commons Attribution 4.0 International License.

Read Full License 


\title{
MBLDP-R: A Multiple Biomolecules based Rapid Life Detection Protocol Embedded in a Rover Scientific Subsystem for Soil Sample Analysis
}

\author{
Akib Zaman ${ }^{1}$, Fardeen Ashraf ${ }^{1}$, Haseena Khan ${ }^{2}$, Faria Noshin Ahona ${ }^{2}$, Oiliullah Samir ${ }^{3}$, Asif Mahmud \\ Rayhan $^{3}$, Sadia Nur Nazifa ${ }^{1}$, Hafsah Mahzabin Chowdhury ${ }^{1}$, Md. Mahbubur Rahman*1 \\ ${ }^{1}$ Department of Computer Science and Engineering, Military Institute of Science and Technology, Mirpur \\ Cantonment, Dhaka 1216, Bangladesh. \\ ${ }^{2}$ Department of Biochemistry \& Molecular Biology, University of Dhaka, Faculty of Pharmacy, Curzon \\ Hall Area College Road, Dhaka 1000, Bangladesh. \\ ${ }^{3}$ Department of Mechanical Engineering, Military Institute of Science and Technology, Mirpur \\ Cantonment, Dhaka 1216, Bangladesh.
}

\begin{abstract}
A rapid multiple biomolecules based life detection protocol (MBLDP-R) from soil samples is proposed to embed in a scientific rover subsystem targeted for planetary analysis missions complying the guidelines of Science Mission of University Rover Challenge 2021 (URC 2021). The proposed protocol selects suitable biomolecules from a preliminary list through a requirement analysis driven filtration process emphasizing two factors: a) rules of URC 2021 and b) compatibility of the biomolecule test equipment to be embedded in a rover subsystem. To sort out the best test methods for finally selected biomolecules, a weighted qualitative test scoring methodology is applied. A rover subsystem that implements the protocol was built to perform onboard sample analysis. Evaluation results show that: 1) ) the proposed MBLDP-R protocol could effectively predict the classes with an average f1-score of $98.65 \%$ (macro) and $90.00 \%$ (micro) and the area under the Receiver Operating Characteristics (AUC-ROC) curve shows the sample categories to be correctly predicted $92 \%$ of the time (97\% Extant, $88 \%$ Extinct and $92 \%$ in case of NPL) and 2) the protocol is time-efficient with an average completion time of 17.60 minutes that demonstrates the rapid nature of the protocol to detect bio signatures in soil samples.
\end{abstract}

Keywords: Life detection, Multiple biomolecule integration, Qualitative test-scoring, Life-detection rover subsystem, Computer-aided analysis. 


\section{Introduction}

Biomolecules are direct products of any cell or living organisms. Soil, also known as pedosphere, serves as a habitat for various life forms and is a rich source of organic matter ${ }^{1}$. Moreover, biomolecules are important components to test for the presence of life $^{2}$. Studies ${ }^{3-6}$ have revealed the efficiency of a single type of biomolecule such as protein, nucleic acid etc. as the detection method for biosignature. Additionally, most of them ${ }^{7-9}$ are based on a theoretical framework and have not been implemented for ground tests. However, a biosignature detection protocol using multiple biomolecules can be more efficient and optimal in this regard.

To promote innovation of life detection technologies in Mars exploration, Science Mission is included as one of the four missions that participating teams are required to perform at the University Rover Challenge (URC), one of the premium global Robotics Challenge organised by the Mars Society at the Mars Desert Research Station (MDRS), Southern Utah, USA. This mission demands a time-bound, accurate, innovative and on-site biosignature detection protocol that needs to be implemented as a rover subsystem. The team qualified for the final round with an outstanding score of $92.78 \%$ (15.50 out of 16 in Science Mission Preparedness). While solving this Science challenge, the team researched the integration of various biomolecules for optimizing the detection of biosignature maintaining the constraints of the competition and developed the required rover subsystem to succeed in the mission.

The objective of this research can be enumerated as a) development of an efficient and rapid on-site lifedetection protocol from soil samples integrating multiple biomolecules; b) development of a mechanical rover subsystem for soil sample collection and classification for implementing the proposed biosignature detection protocol and c) proposing a novel evaluation methodology to assess the performance of the protocols that are used for biosignature detection from soil samples. The outcome of this research can be useful in exploring the integration of multiple biomolecules in the case of planetary analysis. Assuming the signatures of life on Mars are made of similar biomolecules that exist on Earth, the proposed protocol in this research can be a practical tool to implement in subsequent Mars exploration missions.

This paper is divided into seven sections. The following section, section 2 describes the prior work in this field. The research methodology is described in section 3. The development of the protocol is described in section 4. Thereafter, the development of the rover subsystem for scientific exploration is described in section 5. Evaluation of the samples using real-life samples is described in section 6 . Finally, section 7 summarizes the contribution of this work along with the limitations and the future scopes.

\section{Literature Review}

Several studies have been conducted to find the presence of life in soil samples. Some of these are directly intended for the exploration of Mars and the others have focused on finding a generic solution for the detection of life. Recko et. al. ${ }^{10}$ developed a robotic platform-based soil sample collection technique with the development of a unique sample collection method and suitability test based on humidity and UV light observations before collecting the sample. However, the study did not cover the possible methods of soil sample analysis for the detection of biosignature. Neveu et. al. ${ }^{8}$ proposed a theoretical framework to guide the design of investigations to detect microbial life within the practical constraints of robotic space missions. They discussed the extraction of features related to life detection and the methodology for detecting them. However, this basic framework simply assumed the probability of the presence of microbial life to be either above or below a rejection threshold and in a realistic scenario, this measurement can be improved by quantifying a set of distinct numerical probability values. Kite et. al. ${ }^{7}$ also described a framework with a proposition that scientific value is maximized if life-detection missions are flown as hypothesis tests where a negative detection can also become scientifically valuable. Similarly, Sharukh et. 
al. ${ }^{9}$ discussed a theoretical framework for emphasizing the importance of the companion mini-rover system for deep narrow scientific investigations. The results of these proposed studies are based on the theoretical framework or hypothesis which can largely be explored with the development of robotic subsystems to evaluate any protocol with empirical datasets.

On the contrary, Kiflen et. al. ${ }^{5}$ developed a UV-C spectrometer based on Ribonucleic Acid (RNA)/ Deoxyribonucleic Acid (DNA) extraction method to detect nucleic acid in the soil sample. On a similar note, Goordial et. al. ${ }^{4}$ has used a mixture of multiple low-cost techniques for the detection of viable extant microorganisms and nucleic acid from the soil sample in a environment. Similarly, Mojarro et. al. ${ }^{11}$ developed an automated in situ life-detection instrument from soil sample that integrates nucleic acid extraction and nanopore sequencing: the Search for Extra-Terrestrial Genomes (SETG) instrument to isolate and determine the sequence of nucleic acids from extant or preserved life on Mars. However, automation of these multi-steps incorporating sophisticated key steps for successful DNA extraction such as desalting, competitive binding, DNA-protein separation etc. is resource exhaustive and costly. In another study, Mora et. al. ${ }^{6}$ created a Microchip Electrophoresis Laser-Induced Fluorescence (ME-LIF) instrument to receive a liquid sample of soil and analysed it in an automated module. This was a foundational development of microchip electrophoresis instruments for potential life-detection missions. However, this automated module was developed to evaluate liquid soil samples based on the detection of protein (amino acid). Similarly, Abrahamsson et. al. ${ }^{3}$ in their study have prominently marked amino acid as a significant bio-molecule for detection of life and proposed a novel automated chiral amino acid analysis method. These studies described various individual techniques of soil sample analysis.

\section{Methodology}

The methodology of this research has been shown in Fig. 1. At first, all the possible important biomolecules such as protein, carbohydrate, nucleic acid, ammonia, pigment and lipid are listed. The listed biomolecules are filtered to meet the URC 2021 guidelines. Then, a protocol is developed through an extensive study of correlation among the selected biomolecules. Among all the popular methods for the detection of the selected biomolecules, a weighted qualitative test scoring is made to reveal the best test methods for each of the biomolecules. Finally, the best test methods are implemented and integrated with the scientific exploration subsystem for the constructed rover PHOENIX (Fig. 4a). An exhaustive evaluation was carried out using empirical soil samples to evaluate the performance of the proposed protocol.

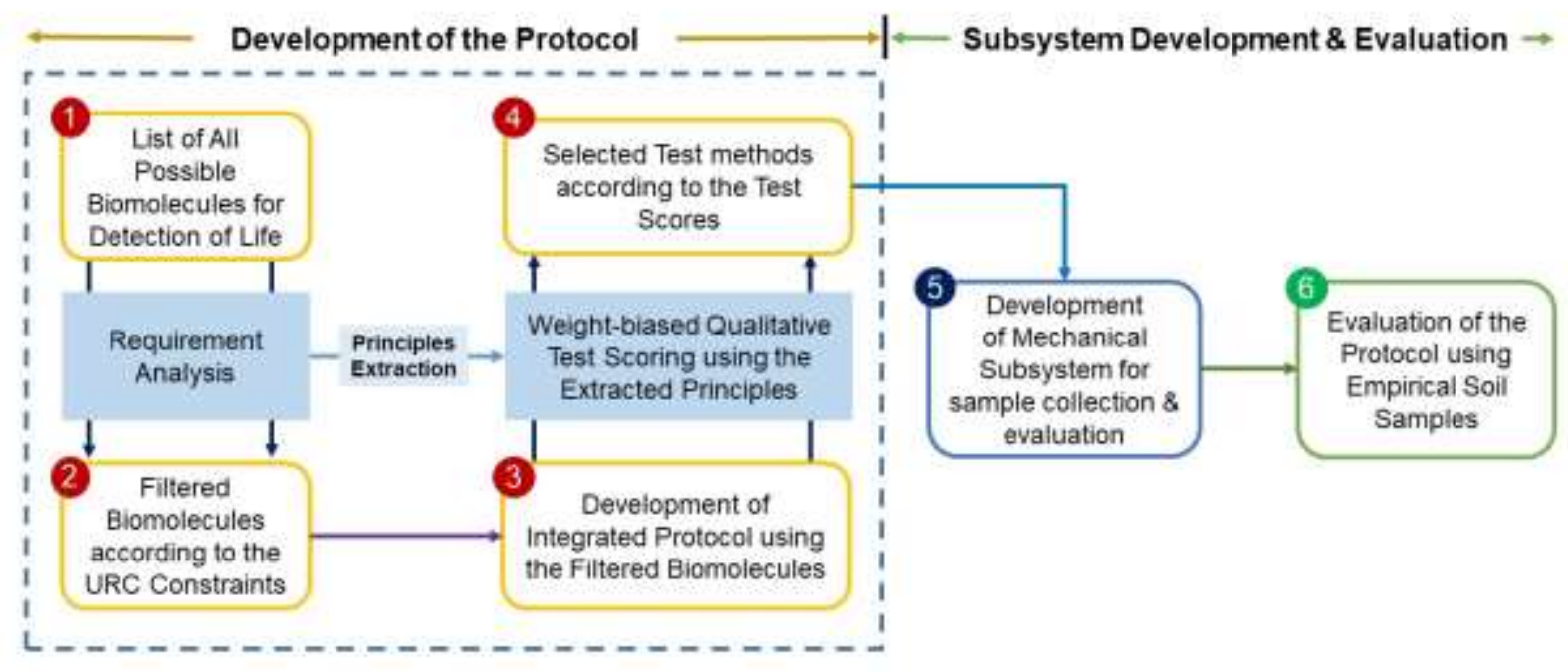

FIG. 1. Research framework. 


\section{Development of the Protocol}

The multiple biomolecules-based life detection protocol; MBLDP-R was developed with the accumulation of works conducted in 4 phases as 1) Potential list of biomolecules 2) Selection of biomolecules based on requirement analysis 3) Development of the structure of the protocol. 4) Qualitative test scoring for selection of the best test methods.

\subsection{Phase 1: Potential list of biomolecules}

For making the initial list of the biomolecules: protein, carbohydrate, ammonia, nucleic acid, lipid and pigment are considered as potential biomolecules for creating the multiple biomolecules based biosignature detection protocol. Since ammonia is derived from metabolism of amino acids and other biomolecules which contain nitrogen and is produced in soil from bacterial processes it is included in the potential list of biomolecules.

4.1.1. Protein. Extensive research circling archaeological remains dating from roughly 6000 years from today have pointed towards the correspondence between bimolecular preservation and the relationship between proteins, primarily collagen, and mineral components, the durability of which depends chiefly on the elements of the soil ${ }^{12}$. If the thermal history of the burial environment is considered the deciding factor, relevant studies and models suggest that from warmer environments, proteins may provide opportunities to recover genetic information, as the diagenesis is controlled by slow, chemical decay of the organic phase ${ }^{13}$.

4.1.2. Ammonia. Mineralization of nitrogen takes place in two steps, namely, ammonification and hydrolysis. In the first step, organic nitrogen is converted to ammonia, which in turn converts to ammonium ions in the presence of water in the second step. Ammonia once formed, quickly escapes from the soil into the air. The positively charged ammonium ion is attracted to the negatively charged soil particles and thus acts as an exchangeable cation in soil ${ }^{14}$. The process is given below:

First step: Ammonification of organic nitrogen

$$
\begin{gathered}
\mathrm{R}-\mathrm{NH}_{2}+\mathrm{H}_{2} \mathrm{O} \rightarrow \mathrm{NH}_{3}+\mathrm{R}-\mathrm{OH}+\text { energy } \\
\text { Second step: } \mathrm{Hydrolysis} \text { of ammonia } \\
\mathrm{NH}_{3}+\mathrm{H}_{2} \mathrm{O} \rightarrow \mathrm{NH}_{4}^{+}+\mathrm{OH}^{-}
\end{gathered}
$$

4.1.3. Carbohydrate. Carbohydrates play a key role in biological recognition processes ${ }^{15,16}$. Carbohydrates can be classified as monosaccharides, oligosaccharides and polysaccharides. Monosaccharides such as galactose and mannose are the chief forms of carbohydrates produced by microorganisms, while arabinose and xylose are formed by plant tissue and roots ${ }^{17,18}$. These carbohydrates are the byproducts of microbial metabolism and have vital roles to play in the formation and stabilization of soil structure ${ }^{13}$.

4.1.4. Nucleic Acid. Nucleic acids are considered an important mark of living cells and are the basic molecules of life ${ }^{19}$. They are naturally made polymers consisting of nucleotides that can store, encode, transmit and express genetic information ${ }^{20,21}$. The first demonstrations of the existence of deoxyribonucleic acid (DNA) in archaeological remains are found in a report by $\mathrm{Pabo}^{22}$ in which DNA was recovered from a mummified Egyptian child (radiocarbon dated to nearly 2,500 years). This further strengthens the selection of nucleic acid for the consideration of extinct or extant life.

4.1.5. Pigments. Pigments, lignin and other polymeric components are found in high amounts in plants ${ }^{23}$. The largest two sources of pigments are plants and microorganisms ${ }^{24,25}$. Bacterial pigments can be obtained from soils that are harvested commercially for a range of purposes including their use in 
medical, food, cosmetic and textile fields ${ }^{26}$. These pigments, which are responsible for the appearance of colours in higher plants are classified into several groups: chlorophylls, carotenoids (carotenes, xanthophylls), flavonoids (chalcones, anthocyanins, flavones, flavonols) and betalains (betaxanthin, betacyanin $)^{27}$. Moreover, in prokaryotic (cyanobacteria) and eukaryotic cells (cyanelles, red algae and cryptomonads) a type of water-soluble photosynthetic pigments called phycobilins can be found in the cytoplasm or the stroma of the chloroplast ${ }^{28}$.

4.1.6. Lipids. Lipid is one out of the three most abundant biomolecules found on animal tissues, the other two being protein and carbohydrate ${ }^{23}$. Lipids also make up between 2 and $20 \%$ of dry weights of bacteria $^{29}$. Up to $20 \%$ of soil humus exists in the form of lipids ${ }^{30}$.

\subsection{Phase 2: Selected biomolecules based on requirement analysis}

Requirement analysis is conducted emphasizing two factors: a) rules of URC 2021 and b) compatibility of the test equipment for the biomolecule with the rover subsystem.

In URC 2021 ${ }^{31}$, the Science mission was designed to find out the presence of life in given soil samples. The goal was to categorize the samples into three significant classes as a) Extinct, b) Extant and c) No Presence of Life, where,

1. Extinct: Life that is fossilized and no longer metabolizing ${ }^{31}$.

2. Extant: Life that is metabolizing, or has so recently died that the biomolecules are yet to be degraded $^{31}$.

3. No Presence of life: No existence of life, inanimate/abiotic objects ${ }^{31}$.

URC 2021 set various constraints in the case of the detection of biomolecules. One of the foremost constraints was on the use of hazardous substances like concentrated nitric acid $\left(\mathrm{HNO}_{3}\right)$, sulphuric acid $\left(\mathrm{H}_{2} \mathrm{SO}_{4}\right)$ etc. to conduct biomolecule detection tests. Moreover, the time limitation of 30 minutes for test completion played a major role in the case of selecting the biomolecules. Besides, the rover needs to traverse from the start gate to the sample location, collect the data from the samples, and pass it back to the base station. Thus, an onboard payload with the necessary testing capability (Fig. 5) is required to be developed which will also be adept in sending necessary data back to the base station. Additionally, the weight of the total rover system with the scientific exploration subsystem must be less than $50 \mathrm{Kg}$, which restricts test designs based on heavy equipment. Finally, the budget limit for the whole system was US dollars 18000. An analysis of all six potential biomolecules based on the requirements was made to find out the suitable biomolecules for the protocol.

Traditional spectrophotometric methods of protein detection include the Biuret test, Ninhydrin test, Xanthoproteic test, Millon's test and many more ${ }^{32}$. Although some of these traditional methods are not feasible considering time constraints and test preparations, some methods such as Biuret, Ninhydrin, and Xanthoproteic tests can be done under a time range of 1 to 10 mins while applying a water bath as a heating source under a temperature range of 60 to 100 degrees $^{32,33}$. More modern methods of protein detection include the use of various mass spectrometers such as IT-LIT, Q-Q-ToF, ToF-ToF, FT-ICR, Q-Q-Q, QQ$\mathrm{LIT}^{34}$. However, considering the factors of requirement analysis such as the complexity of the equipment required for the tests, compatibility with the rover system and budget constraint of URC 2021, the traditional methods seemed to be more suitable than the modern methods. On a similar note, methods of detecting ammonia from soil samples may include Schloesing's methods, Baumann's method or Russel's methods ${ }^{35}$. Ammonia can also be detected by using dilute sodium hydroxide solution and heating it. The resultant ammonia gas can be detected by its distinct pungent smell or by using a damp red litmus paper, which turns blue if the gas is present. This method is both simple and fast based on the type of heating source (less than a minute for direct heat). At present, various types of ammonia gas detecting sensors are 
available such as metal oxide-based sensors, conducting polymer sensors, tunable diode laser absorption spectroscopy (TDLAS), electrochemical sensors, surface acoustic wave sensors, field-effect transistor (FET) sensors etc ${ }^{36}$. Easy detection procedures and readily available sensors make ammonia a potential biomolecule in these circumstances. On the other hand, for identifying the presence of carbohydrate in general or its various groups, several rapid tests based on specific colour reactions are available such as Molisch's and Anthrone tests for carbohydrates in general, Iodine test for glycans (starch, glycogen), Seliwanoff's test for ketoses, Fehling's test, Benedict's test and Picric acid test for reducing sugars, Mucic acid test for galactose, Bial's test for pentoses and Barfoed's test to distinguish between monosaccharides from reducing disaccharides ${ }^{37}$. The majority of these test methods are fairly less complex and also timeefficient. Additionally, the equipment for conducting these tests is simple, lightweight and compatible with the rover system. Based on the conditions of requirement analysis, all three biomolecules: carbohydrates, ammonia and protein have the essential characteristics to be integrated as potential candidates for the protocol.

On the other hand, before the analysis of nucleic acid (DNA, RNA) from soil samples, the soil material must be removed; the nucleic acids or the microbial cells must be extracted from the soil particles and humic substances that will otherwise obscure almost any observation ${ }^{38}$. This multistep approach of detection methods further complicates the implication of the nucleic acid detection system embedded in the rover. Moreover, automation of these multi-steps incorporating sophisticated key steps for successful DNA extraction such as desalting, competitive binding, DNA-protein separation etc. is resource exhaustive and costly. In addition, extraction of DNA from different sources like a spore is generally a time-consuming process employed in the detection of nucleic acids from Martian or Mars-like soils as well ${ }^{11}$ and biological reagents used in this process are a concern in the context of URC protection regulations. Moreover, $30 \mathrm{~min}$, the time set for the competition is not enough for the rover to manoeuvre, collect the sample and use the DNA extraction kits for sample analysis. In the case of pigment detection, paper chromatography ${ }^{39,40}$ is a prominent traditional method. Although the method can be used for various types of pigment detection, a multi-step procedure increases its complexity as an onboard testing mechanism on the rover subsystem. Additionally, modern approaches include the use of High-Performance Liquid Chromatography (HPLC) which can separate photosynthetic pigments such as chlorophylls, carotenoids etc ${ }^{41-43}$. is also a complex multi-step procedure comprising pigment extraction and HPLC analysis. The equipment of HPLC is sophisticated, non-modifiable and very costly. A portable HPLC machine weighs up to $8 \mathrm{Kgs}^{44}$, which is not very efficient considering the $50 \mathrm{Kg}$ weight limit of the total rover system. Thus, it is certainly not possible to include pigment detection in the multiple biomolecule-based protocol. On the other hand, conventional methods for lipid quantification rely on solvent extraction and either gravimetric assays or chromatographic determination, which are time-consuming multi-step procedures that depend on the creation of the required test environments ${ }^{45}$. Thus, the complexity level of these methods is not suitable for the rover system and it is not convenient for executing the methods in an external environment. In contrast, colourimetric methods for lipid quantification constitute an attractive choice due to their fast response and simplified sample handling; however, they also usually require other preliminary steps such as cell disruption and lipid extraction ${ }^{46}$ that is not convenient based on the compatibility and complexity. Furthermore, other nonconventional spectroscopic methods include infrared spectroscopy, nuclear magnetic resonance spectroscopy, Raman spectroscopy, fluorescence spectroscopy and dielectric spectrometry ${ }^{46}$ which involve costly equipment. The above analysis shows that the tests of nucleic acid, pigment and lipid detection do not fulfil the minimum criteria of the requirement analysis. Thus, they were not considered for further analysis. Based on the above-mentioned discussions, protein, carbohydrate and ammonium ions were selected to develop multiple biomolecules based biosignature detection protocol from soil samples.

\subsection{Phase 3: Development of the structure of protocol}


Extracted information of the selected biomolecules is utilized to create the structure of the proposed protocol. In the three-layered decision-tree based architecture of the protocol the presence of protein is checked in the first layer followed by the carbohydrate and lastly ammonium ion. There are eight possible outcomes of the proposed protocol as shown in Fig. 2. The presence of all three types of biomolecules is classified as Extant and the absence of all three types gives a result of NPL. Proteins are found in all living organisms and are not found abiotically. Moreover, protein is also a notable type of biomolecule that can be found in fossils buried underground ${ }^{13}$. On a similar note, carbohydrates can be found in the soil samples because of microorganisms, plant tissue or tree roots ${ }^{47}$. Carbohydrates can also be found on fossil samples belonging to specimens of protozoa, mollusca, arthropoda and plants ${ }^{48}$. Moreover, ancient sedimentary rocks are also a source of carbohydrates ${ }^{49}$. Although both proteins and carbohydrates can be found as fossil molecules, in terms of decay resistance carbohydrate is ahead of protein which indicates that the possibility of carbohydrate preservation in fossil records or incorporation into fossil fuels is more than protein ${ }^{50}$. For this reason, regardless of the other two types of molecules, the presence of protein will always give us a result of Extant. When protein is absent, the framework will prioritize on the presence of carbohydrate. If carbohydrate is present despite the absence of protein, the framework will always give a result of 'Extinct', regardless of the presence of ammonium ion (NH4+). Although ammonium ion (NH4+) can be present in living organisms, it can be produced abiotically or biotically ${ }^{51}$. For this reason, only the presence of ammonium ion cannot specifically indicate any of the three results (Extant, Extinct and NPL). To avoid complications, this uncertain result is considered as NPL by the framework.

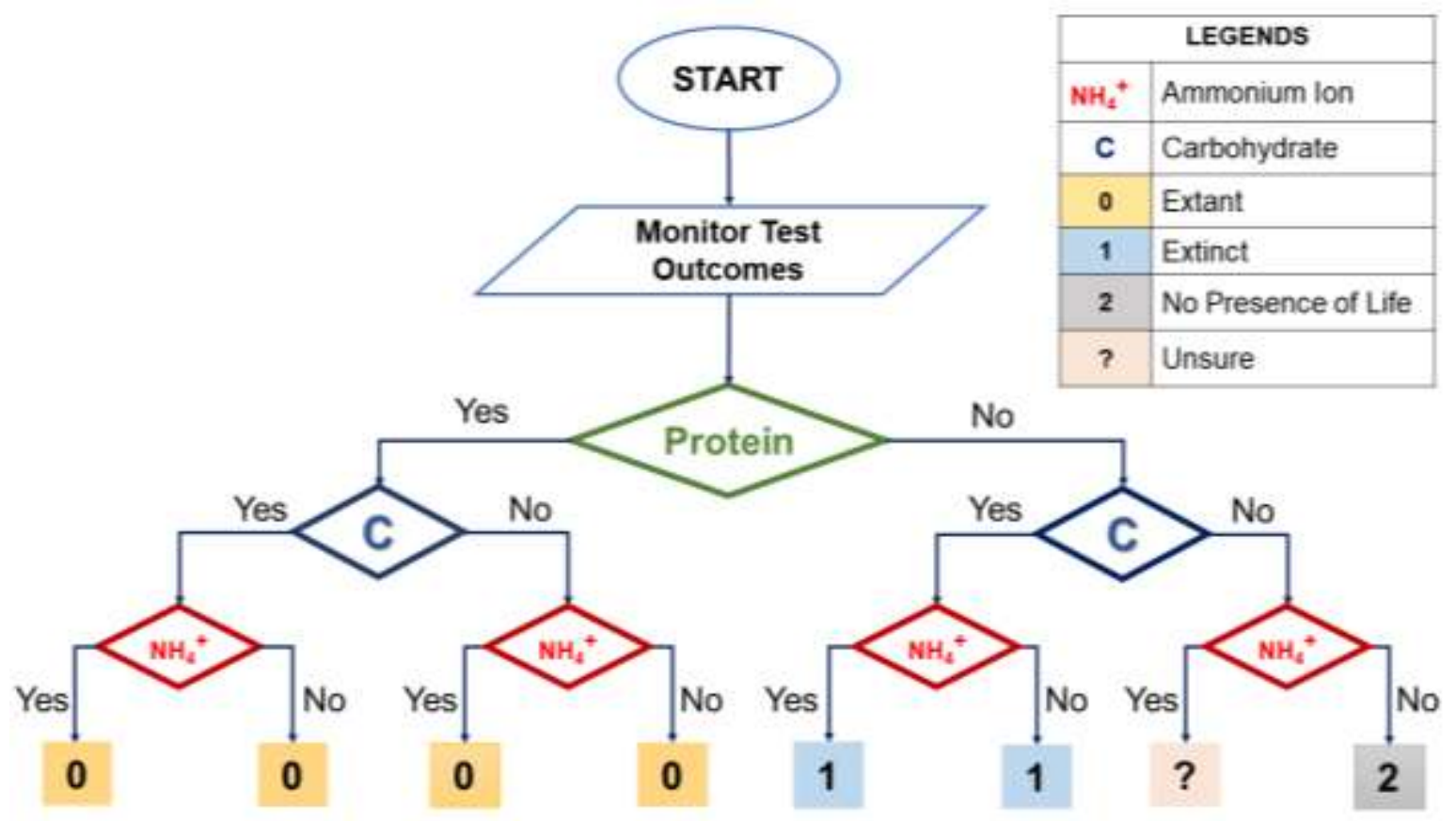

FIG. 2. Structure of the multiple biomolecule-based framework for soil sample detection.

\subsection{Phase 4: Qualitative test scoring for selection of test methods}

Various tests exist for detecting proteins, carbohydrates and ammonium ions. However, the abovediscussed requirement analysis does not allow all the variants of the tests to be conducted in an onboard laboratory as a rover subsystem. Thus, six principles were extracted from the requirement analysis and the principles assigned a numerical weight according to priority based on requirement analysis factors. After that, a weighted test scoring based on these principles was conducted to select the best suitable test methods for the different biomolecules. Selected principles for qualitative analysis were as follows: 
4.4.1. Hazardous Substances (HS). Any substance that can potentially affect safety and cause health hazards is deemed hazardous. Properties that can compromise the safety of the users and the components include flammability, explosiveness, toxicity, and the ability to oxidize. Concentrated $\mathrm{H}_{2} \mathrm{SO}_{4}$, $\mathrm{HCl}, \mathrm{NaOH}, \mathrm{H}_{2} \mathrm{O}_{2}, \mathrm{Br}$ are some examples of hazardous substances. For strict safety purposes, hazardous substances are not allowed in the on-site tests (Labels: Require HS-0, Don't Require HS-1; Weight = 1).

4.4.2. Time (T). The amount of time required to conduct an experiment and to get the result (Labels: > than 25 mins-0, $<=25$ mins -1 , Weight=0.9).

4.4.3. Colour Identification $(\mathrm{Cl})$. It defines the recognition of colours of the components of a chemical reaction once it reaches the desired stage. Although colour identification alone is not a reliable procedure in the identification of compounds due to the effect chemical impurities have on the colour of compounds, this technique essentially provides a head start to the identification process (Labels: Distinct colour-1, No Distinct Colour-0, weight =0.8).

4.4.4. Unit Compound Identification (UCI). Unit compound (UC) identification such as amino acids for proteins and monosaccharides for carbohydrates is important as it ensures the feasibility of the test for all variants of that particular biomolecule (Labels: UC identified-1, UC Not Identified-0, weight $=0.7)$.

4.4.5. Direct Heat Contact (DHC). Direct heat contact refers to the process of test completion over a heating component rather than using a water bath. Although a water bath can provide more precise control over the heat generated for the tests, DHC is more suitable for a rapid and spill-free approach. Usage of heating components with direct contact of testing beaker also removes the complications of using water bath heating mechanism on a rover body. Regarding the safety of the rover non-flame generating heating, components are considered for DHC. Thus, it is more convenient to use direct heat using a heating pad for a rover subsystem (Labels: Functional under DHC-1, Not Functional under DHC-0, weight =0.5).

4.4.6. False Positive (FP) (0.3). While identifying a specific compound, the presence of certain compounds can cause a positive result while the actual compound is absent. This positive result turns out to be a False Positive that is not desired (Labels: Doesn't Give False Positive -1, Gives False Positive - 0, weight $=0.3$ ).

Modern instrumental methods are far more sensitive and accurate in terms of detecting or quantifying certain biomolecules than traditional spectrophotometric methods. However, these modern instruments can be expensive and difficult to manipulate for certain mission scenarios as discussed in the previous subsection. Considering the budget constraints given by the URC and the feasibility of detecting these biomolecules on rover bodies through on-board analysis, mostly traditional spectrophotometric detection methods have been considered in this research. Some of the most popular test methods for the detection of proteins, carbohydrates and ammonium ions are considered for the scoring procedure. The tests are scored according to the characteristics of the test procedures in the six selected scoring principles. They are Biuret, Xanthoproteic, Millon's and Ninhydrin test for protein detection; Benedict's, Seliwanoff's, Barfoed's, Molisch's and Iodine/Lugol's tests for carbohydrate detection; and Schloesing's method, direct distillation with magnesium ion and Litmus strip tests for ammonium detection. The above-mentioned tests were conducted in the Chemistry laboratory of MIST and the results were documented to determine the score of the principles. Test scoring results of the mentioned methods are shown in Table 1. The total score for any test has been calculated using the formula:

$K=$ Set of Principles $=($ HS $, \mathrm{T}, \mathrm{CI}, \mathrm{UCI}, \mathrm{DHC}, \mathrm{FP})$

$E(K)=$ Label of any test $\mathrm{E}$ for principle $\mathrm{K}[\mathrm{e} . \mathrm{g} .:$ Xanthoproetic $(H S)=0$ ] 
$W_{K}=$ Weight of principle $\mathrm{K}$

Total score for any test $E=\sum_{K}^{K=6} E(K) * W_{K}$

TABLE 1. Weighted Test Scoring of the Biomolecule Detection Methods (X means insignificant).

\begin{tabular}{|c|c|c|c|c|c|c|c|c|}
\hline Biomolecule & Test Name & $\begin{array}{r}\text { HS } \\
\text { (1) }\end{array}$ & $\begin{array}{c}\mathrm{T} \\
(0.9 \\
)\end{array}$ & $\begin{array}{c}\text { CI } \\
(0.8)\end{array}$ & $\begin{array}{l}\text { UCI } \\
(\mathbf{0 . 7})\end{array}$ & $\begin{array}{l}\text { DHC } \\
(0.5)\end{array}$ & $\begin{array}{c}\text { FP } \\
(0.3 \\
)\end{array}$ & $\begin{array}{l}\text { Total } \\
\text { Score }\end{array}$ \\
\hline \multirow{4}{*}{ Protein } & Biuret & 1 & 1 & 1 & 0 & 0 & 0 & 2.7 \\
\hline & Xanthoproteic & 0 & 1 & 1 & 1 & 1 & 1 & 3.2 \\
\hline & Millon's & 1 & 1 & 1 & 0 & 0 & 0 & 2.7 \\
\hline & Ninhydrin & 1 & 1 & 1 & 1 & 1 & 1 & 4.2 \\
\hline \multirow{5}{*}{ Carbohydrate } & Benedict Solution & 1 & 1 & 1 & 1 & 1 & 1 & 4.2 \\
\hline & Seliwanoff's & 0 & 1 & 1 & 0 & 0 & 1 & 2.0 \\
\hline & Barfoed's & 1 & 1 & 1 & 1 & 0 & 1 & 3.7 \\
\hline & Molisch's & 0 & 1 & 1 & 0 & 0 & 0 & 1.7 \\
\hline & Iodine/ Lugol's Solution & 1 & 1 & 1 & 0 & 0 & 1 & 3 \\
\hline \multirow{3}{*}{ Ammonium } & Schloesing Method & 0 & 1 & 0 & $\mathrm{X}$ & 0 & 1 & 1.2 \\
\hline & $\begin{array}{l}\text { Direct Distillation with } \\
\text { Magnesium ion }\end{array}$ & 1 & 0 & 0 & $\mathrm{X}$ & 1 & 1 & 1.8 \\
\hline & Litmus Strip test & 1 & 1 & 1 & $\mathrm{X}$ & 1 & 1 & 3.5 \\
\hline
\end{tabular}

It is evident (Table 1) that although the Biuret test can detect the presence of peptide bonds in a substantial peptide bond concentrated protein sample, it fails to detect the unit compound amino acids (\#4 UCI) in the soil specimen. Moreover, ammonium sulfate can often generate coloured complexes or interfere with colour development resulting in false positives (\#6 FP). On a similar note, Millon's test cannot identify the unit compound (\#4), amino acids in the soil sample despite having a quick completion time of 2-3 minutes (\#2 T). Moreover, compounds like salicylic acid and phenolic compounds can give rise to a false positive (\#6) in this method. Both Biuret and Millon's tests achieved a score of 2.7 (Table 1). In contrast, detection of amino acids by both Ninhydrin and Xanthoproteic tests can be stated as indirect protein assay methods. However, the prime component for the nitration reaction in the Xanthoproteic test is concentrated $\mathrm{HNO}_{3}$ which is a hazardous substance (\#1 HS) and requires careful handling. The use of the Ninhydrin test is time-efficient with the detection time only between 3 to 4 minutes (\#2). It can also easily detect amino acids (\#4) indicating the presence of all types of proteins in the soil sample. Thus, the Ninhydrin test was selected with a total test score of 4.2 while the Xanthoproteic test was the nearest one with a test score of 3.2 (Table 1).

In the case of carbohydrate detection, Seliwanoff's reagent contains highly concentrated $\mathrm{HCl}$ (\#1) that may compromise the safety of the rover. Moreover, it detects aldose and ketose but is unable to detect monosaccharides (\#4) and the use of a water bath (\#5 DHC) is a must in this method. Similarly, 
Iodine/Lugol's solution test can only detect polysaccharides (\#4) and cannot identify monosaccharides. Although Barfoed's method can successfully detect monosaccharides (\#4), direct heat contact (\#5) is not convenient for the test. Again, the concentrated sulfuric acid (\#1) required to conduct Molisch's test for the detection of carbohydrates is highly corrosive and is potentially explosive in its concentrated form. In addition, the presence of some organic acids (citric acid, lactic acid, oxalic acid, formic acid etc.) can give false-positive (\#6) results in this method. Benedict's test identifies monosaccharides (\#4) in a soil sample and does not involve any corrosive ingredients (\#1) in the process. Considering all the principles, Benedict's test achieved the highest test score of 4.2 than Barfoed's (3.7), Iodine/Lugol's (3), Seliwanoff's (2.0) or Molisch's (1.7) test (Table 1). Thus, Benedict's test was selected for the detection of carbohydrates.

In the case of ammonium ion detection, the first Schloesing's method requires strong sodium hydroxide. It, therefore, had to be discontinued because this strong alkali even at low temperatures gradually decomposes the organic nitrogen compounds giving rise to ammonium ions. Hydrochloric acid (\#1) needed in the second Schloesing's method removes approximately $60 \%$ to $70 \%$ of the added ammonium ions ${ }^{35}$. This method requires the distillation of the soil directly with magnesia, and the amount of ammonium obtained by distillation is dependent on the duration of the distillation, which is proportional to the amount of water and soil used and, on the amount of heat applied. Therefore, it takes more time (\#2) to get the ammonium ions by this process. Moreover, these two methods do not give coloured identification (\#3 CI) during the test. The use of hazardous substances and the length of time required to obtain the ammonium ions are drawbacks of the first two methods resulting in a test score of 1.2 and 1.8 respectively (Table 1). On the other hand, in the Litmus Strip test sodium hydroxide solution is mixed directly with the soil sample and then heated. The ammonium ions present in the sample then turns into ammonia gas and this gas turns the red litmus paper blue. With the highest score of 3.5 (Table 1) among all the ammonium ion detection methods, the litmus strip test method was selected for the detection.

\section{Development of the Rover Subsystem}

The rover subsystem has been developed to be attached to the main chassis to provide a tool suitable for collecting soil samples from remote and even hazardous places.

\subsection{Design and fabrication}

For evaluating the proposed protocol, a mechanical payload has been designed as shown in (Fig. 3). The subsystem has four degrees of freedom (DOF) mechanism. There are three main parts of the developed subsystem such as a) manipulator b) sample collection chamber and c) reagent mechanism. The body of the mechanical payload is made of stainless steel and aluminium sheets and was capable of collecting 4 soil samples at a time and conducting tests for protein (Ninhydrin), carbohydrate (Benedict) and ammonium ion (Litmus strip test) concurrently. 


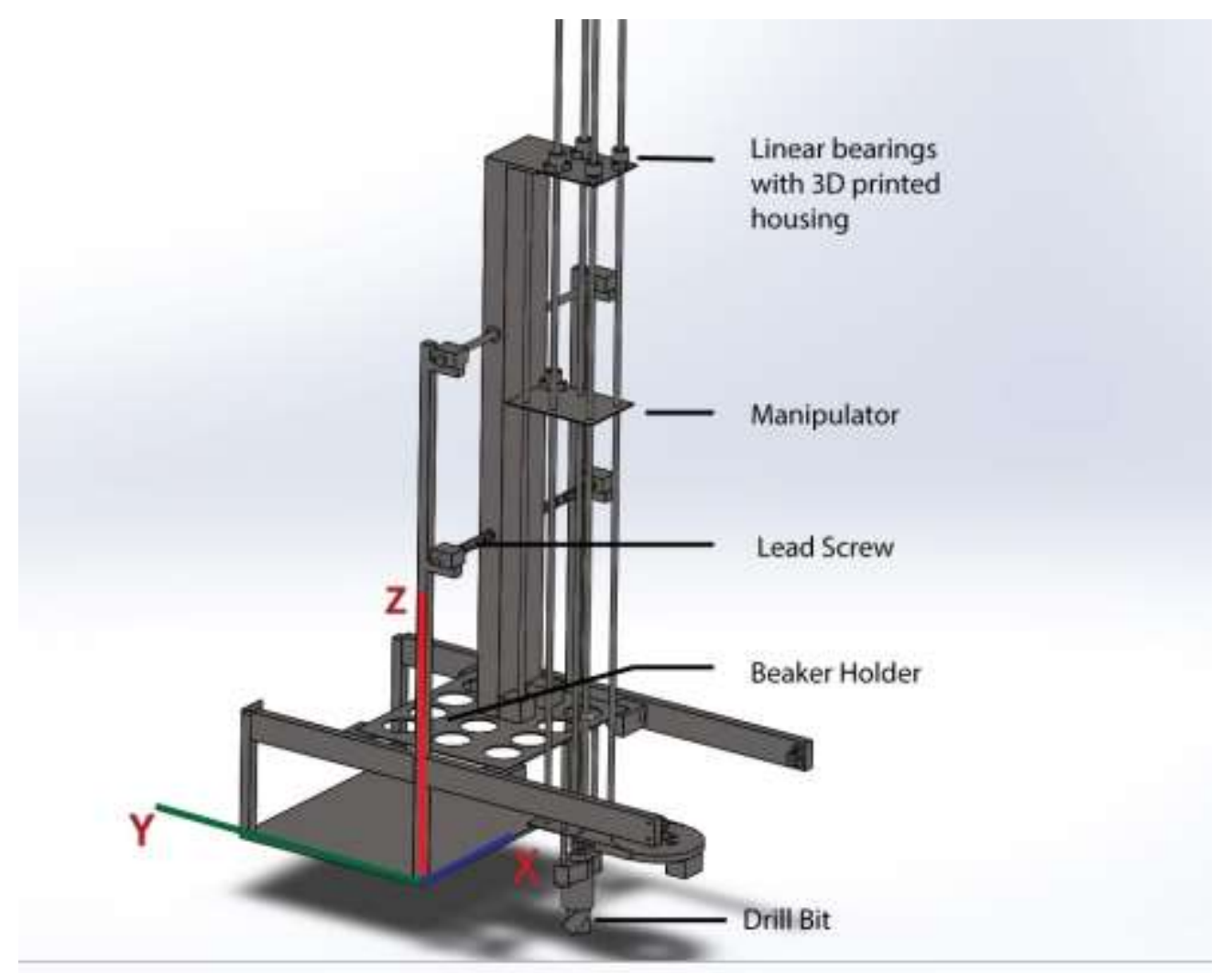

Fig. 3. Design of the rover subsystem.

\subsection{Manipulator}

As shown in Fig. 4b the subsystem is capable of collecting soil samples with three degrees of freedom (DOF) manipulator where a custom-made drill bit has been attached as an end effector. The drill bit is made of mild steel with approximately $100 \mathrm{~mm}$ in length and the outer diameter is $35 \mathrm{~mm}$. Linear bearings with $3 \mathrm{D}$ printed housing are used to ensure smooth motion and linear rods of $8 \mathrm{~mm}$ diameter are used to support the stainless-steel plates. To drive the manipulator, two square threaded lead screws of $8 \mathrm{~mm}$ diameter are used to collect soil samples from the ground and to place it in the beaker for further operation. Two separate stepper motors (NEMA 17) drive the lead screws. A $5 * 8$ coupler connects lead screws and the stepper motors. Using a lead screw mechanism, the manipulator can move along the $\mathrm{X}$ and $\mathrm{Z}$-axes and the end effector rotates along the Z-axis to collect the soil samples. For preventing cross-contamination, a liquid sanitization mechanism is added with the end effector releasing a flow of hydrogen peroxide after each sample collection.

\subsection{Sample collection chamber}

The sample collection chamber consists of 12 (borosilicate glass) beakers, a $2.5 \mathrm{~mm}$ thick aluminiumholding frame and a heat source. The frame alongside the beakers can move back and forth along the Yaxis to collect samples. Samples are arranged along the $\mathrm{Y}$-axis and the concurrent biomolecules tests are planned along the $\mathrm{X}$-axis as shown in (Fig. 5b). A customized heat-pad is used as a heat source capable of generating $3600 \mathrm{Watt} / \mathrm{hr}$ energy for providing necessary heat for the tests. 


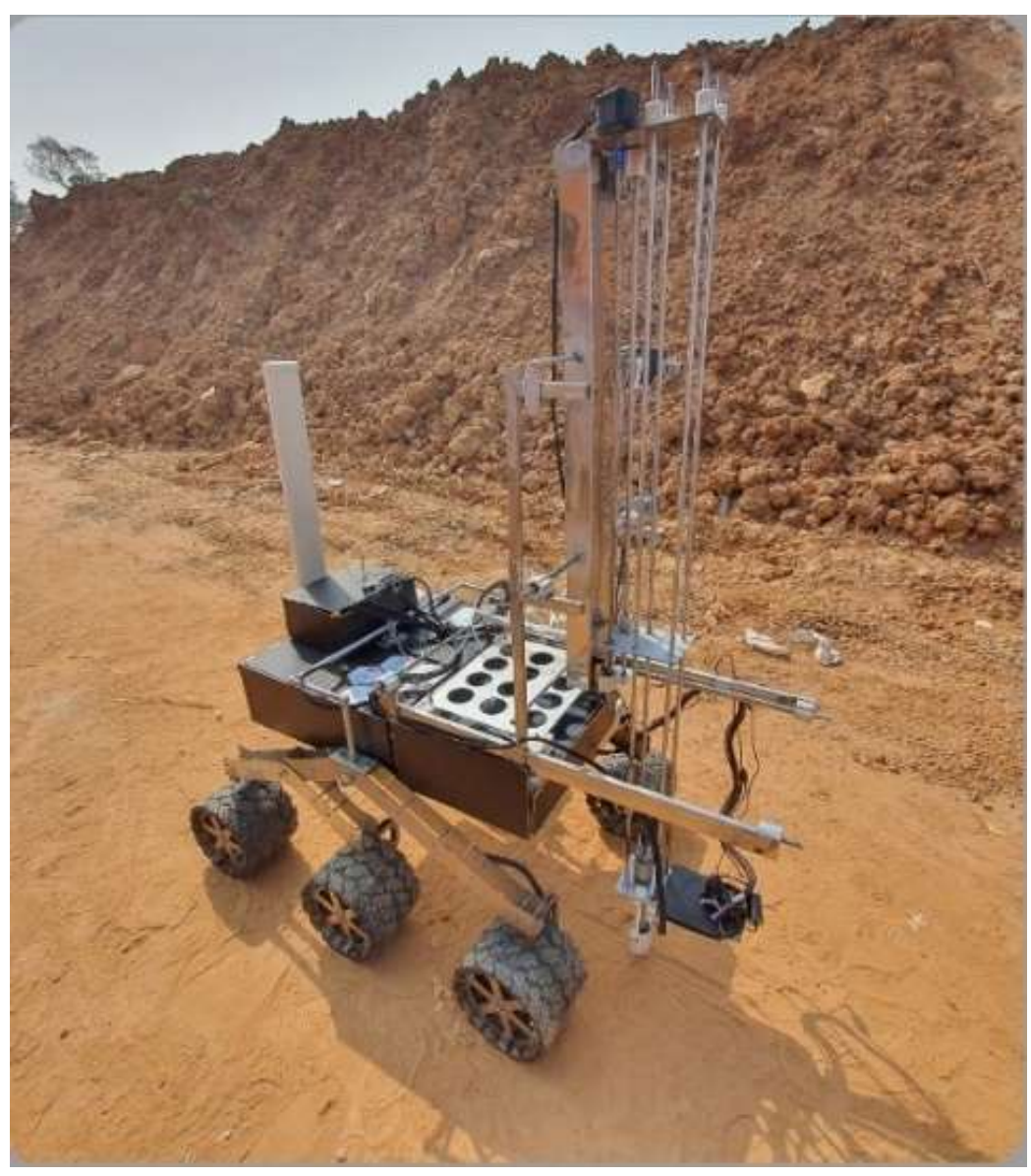

(a)

12 of 24 


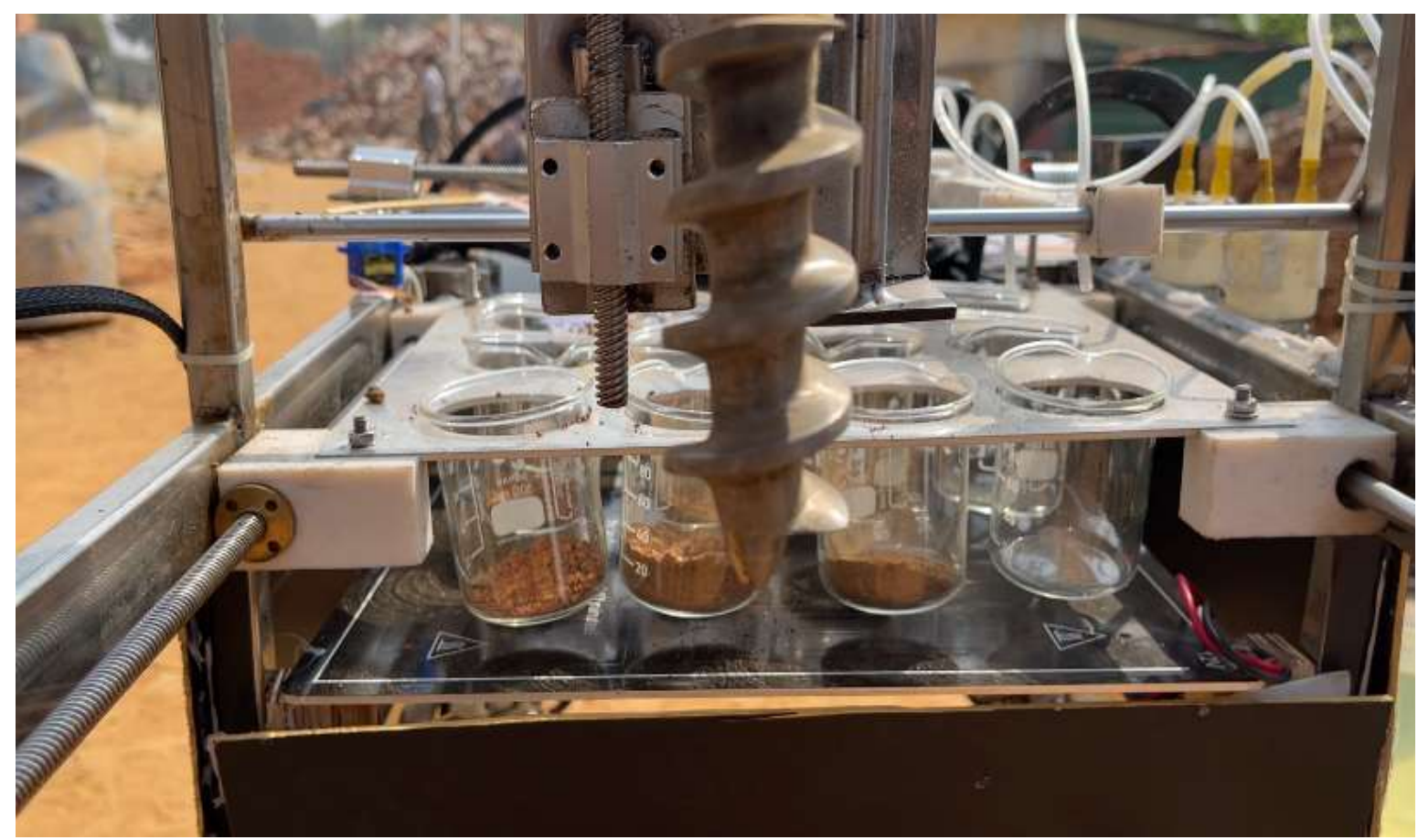

(b)

FIG. 4. (a) Complete rover system (PHOENIX) with the attached scientific subsystem. (b) Collection of soil samples through the subsystem.

\subsection{Reagent flow mechanism}

The reagent mechanism consists of three containers holding the reagents of the selected tests, a litmus mechanism for ammonium test and 1 DOF tube framework. As per the selected tests, the containers are filled with Benedict's reagent (carbohydrate test), Ninhydrin reagent (protein test), Sodium Hydroxide solution (ammonium ion test) and strips of litmus paper (ammonium ion test). Three-12V DC Diaphragm Metering Miniature Short Motor Water Pumps deliver the reagents.

\subsection{Detection mechanism}

Four NEMA 17 stepper motors alongside the drivers were used to mobilize the motions of the subsystem. The whole rover system is controlled manually by a controller remote using a $915 \mathrm{MHz}$ communication frequency, which has a range of more than $1 \mathrm{~km}$. As shown in Fig. 6, the rover at first moves to the desired sample location and collects the sample using the manipulator. Using the motion of the beaker-holding frame along the y-axis, the samples collected are stored in three rows of a dedicated column of the collection chamber. Then, moving the manipulator along the $\mathrm{x}$-axis the rover can collect up to four samples and store them in each column. After collecting samples for all four columns, the reagent flow mechanism adds the regents to the beakers by sliding down the frame along the $\mathrm{x}$-axis for reaching all the columns as shown in Fig. 5a. The heat source provided the required amount of heat. Then, by using a servomotor, a SS bar with three litmus strips was held on top of the beakers of row three as shown in Fig. 5b. The visual colour change of rows one and two and the colour of litmus paper of row three gave the status of protein, carbohydrate and ammonium to the samples respectively. The observation was noted using a USB camera feedback from the base station. Finally, the output of the three results was used as the input to the proposed MBLDP-R to classify the sample. 


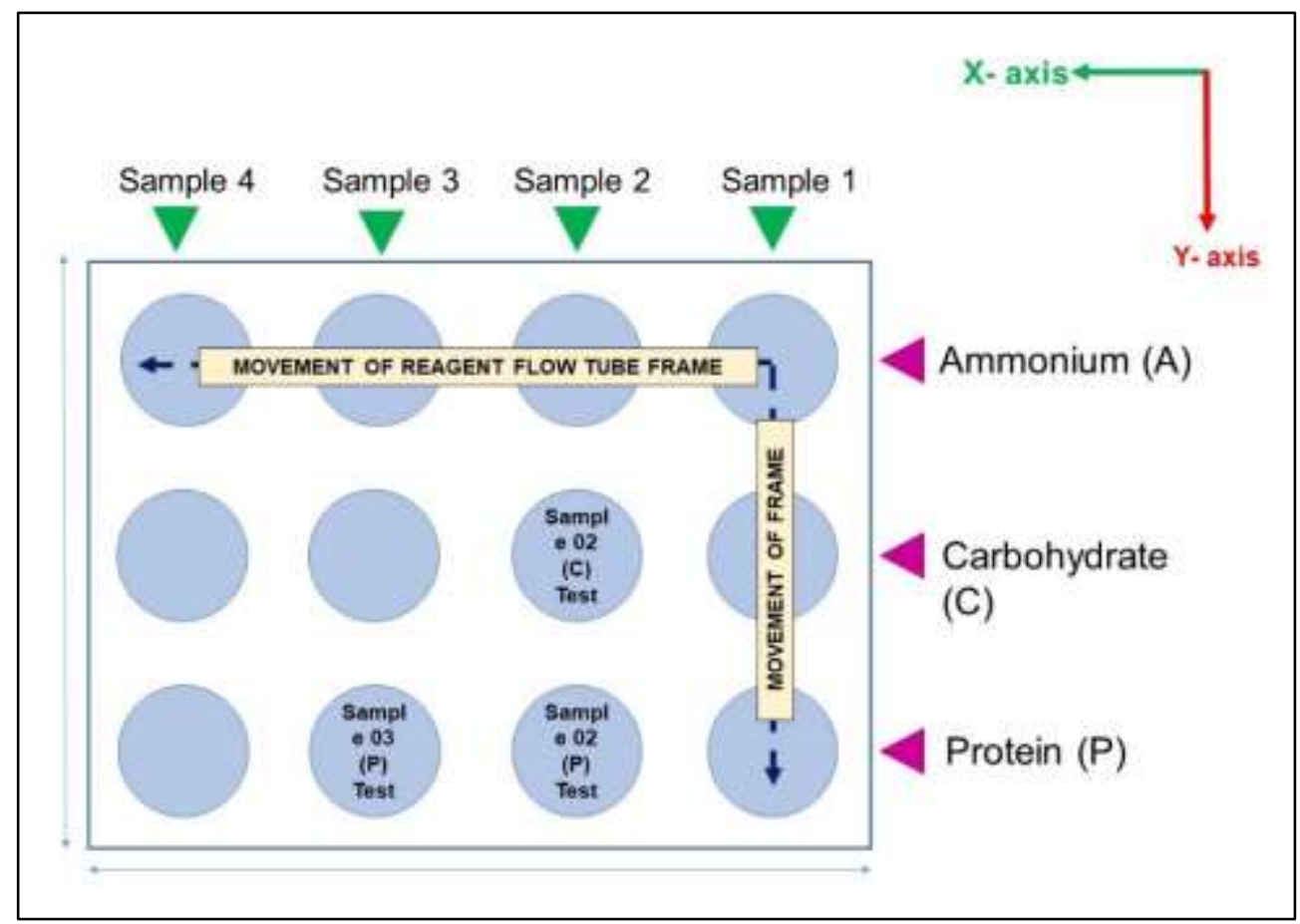

(a)

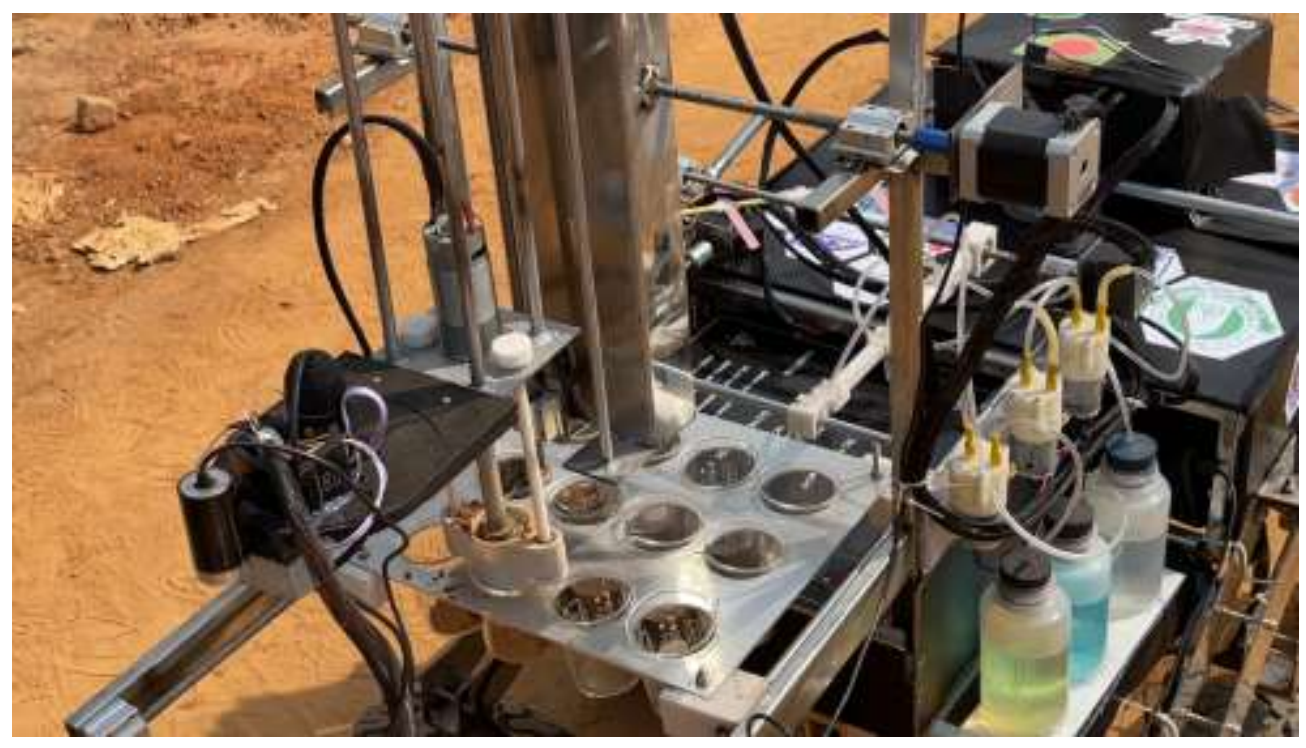

(b)

FIG. 5. (a) Setup of test methods. (b) Onboard sample analysis in the developed subsystem.

\section{Evaluation}

The evaluation study was conducted in the chemistry laboratory of MIST and at a mock science mission field. The tests were conducted in a series of 10 events. At each event, 4 of the samples were placed in various locations of the mock science field. The gathered data from the field were transmitted back to the main base and the samples were predicted using the proposed protocol as a backbone algorithm. The field test videos are available at the deposited official System Acceptance Review (SAR) video ${ }^{52}$ of the team. 


\subsection{Preparation of samples}

The soil samples were collected from a local site. However, the necessary alteration was implemented to increase the bioload or remove it. According to the guidelines of URC 2021, the samples were labelled as Extant, Extinct and No Presence of Life (NPL). The Extant samples were prepared by collecting fertile soil and removing any unwanted rock particles. A few of the samples were modified using extra bioloads (dextrose, albumin, etc.). The No Presence of Life (NPL) samples were prepared by baking the soil for more than 24 hours at 500 degrees centigrade according to the exact guideline of the URC organizing committee $^{31}$. The Extinct samples were prepared in two phases. In phase one, the collected samples were baked for more than 24 hours at 500 degrees centigrade to degrade any type of presence of biosignatures. Then in phase two, fossilized rocks collected from archaeologically significant local sites were mixed with the samples to create an Extinct status.

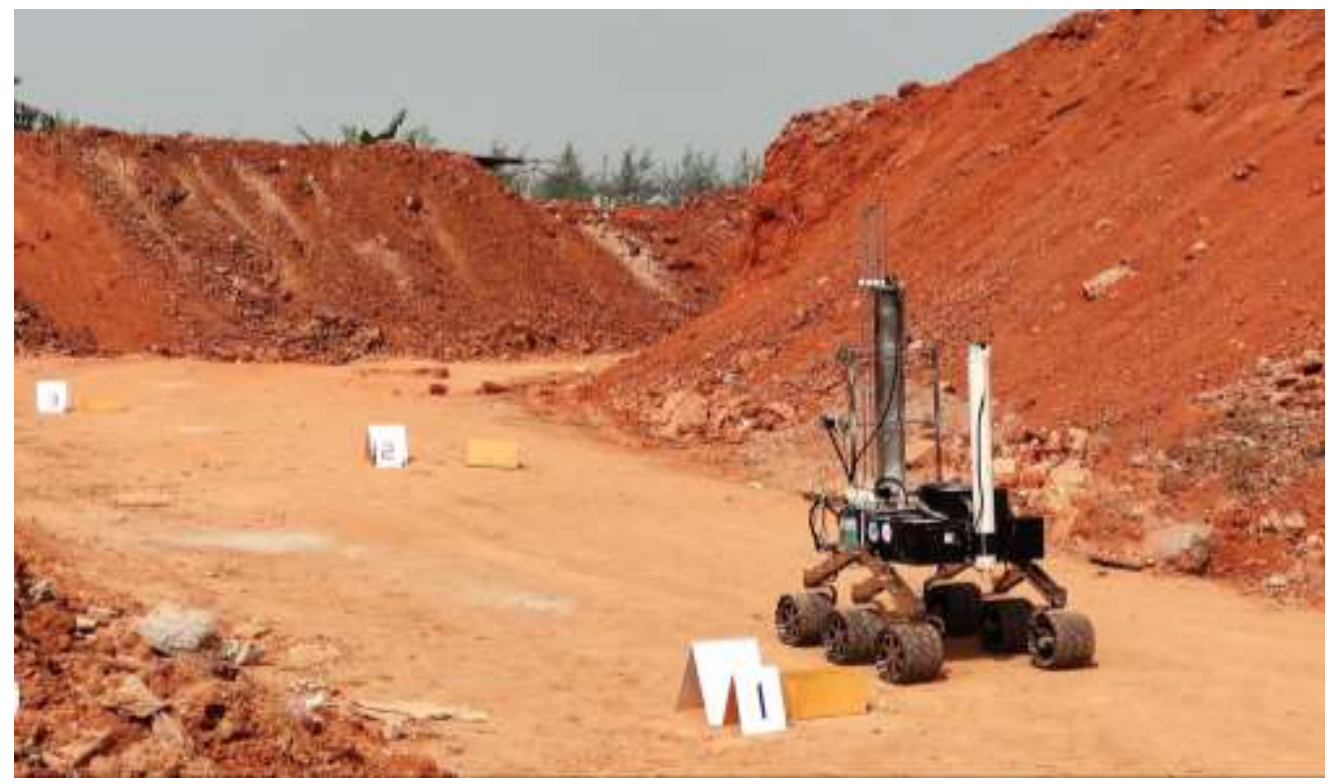

FIG. 6. Evaluation using mock Science Mission setup.

\subsection{Sensitivity Analysis of the Colorimetric Reactions}

An evaluation study was conducted to measure the sensitivity of the tests. The selected Ninhydrin test of this research is sensitive enough to detect up to 0.001 pmol of amines, tested from 15 sets of protein samples. Under the standard conditions of the monitoring assay, this quantity gives a reading at $570 \mathrm{~nm}$ of 0.028 absorbance units and the normal reagent blank is 0.02 units. Amines present less than the lower limit in the soil sample will not produce any colours hence will not be detected by the rover scientific exploration subsystem and hence will produce a False Negative Output.

\subsection{Study procedure}

The evaluation was conducted in a mock science field of MIST. A total of 10 events were conducted where 4 samples were used in each event. In a single event, the rover had to traverse 4 sample locations (Fig. 6) and conduct the onboard sample analysis using the developed subsystem. The evaluation was conducted using 40 samples consisting of 18 Extant, 12 Extinct and 10 NPL samples.

\subsection{Results}

The study found the protocol to be $90 \%$ (36 out of 40) accurate in classifying the samples into desired labels. In the case of Extant samples, the protocol achieved an accuracy of 94.44\% (17 out of 18). For 
Extinct and NPL samples the protocol was found to be $83.33 \%$ (10 out of 12) and 90\% (9 out of 10) accurate respectively. However, accuracy is most suited when single individuals are considered instead of multiple classes $^{53}$. As the evaluation was targeted to identify the performance of the protocol in the case of the mentioned classes, F-score, precision and recall measures were evaluated per class. Performance in identifying classes was also examined through a confusion matrix shown in Fig. 7. Overall classification performance was examined using average measures like micro and macro averaging; these are widely accepted and commonly used in many multiclass classification studies.
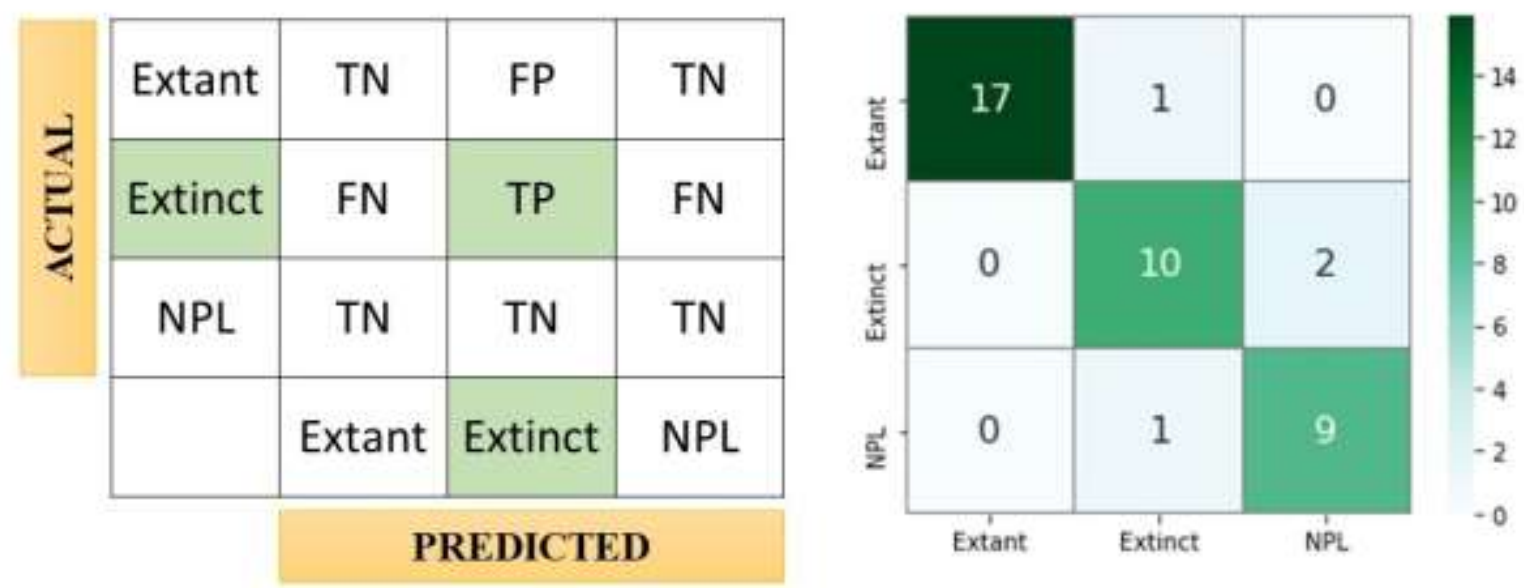

$* \mathrm{TP}=$ True Positive; FP = false Positive; $\mathrm{TN}=$ True Negative; FN= False Negative

FIG. 7. Confusion matrix of the evaluation study of the protocol.

Precision is the fraction of true positive (TP) elements divided by the total number of positively predicted units (column sum of the predicted positives). It expresses the proportion of units the model says are positive and, they are positive. In other words, precision tells how much we can trust the model when it predicts a sample as positive. The recall is the fraction of True Positive elements divided by the total number of positively classified units (row sum of the actual positives). Recall measures the model's predictive accuracy for the positive class: intuitively, it measures the ability of the model to find all the positive units in the dataset. F1-score assesses the classification model's performance starting from the confusion matrix; it aggregates precision and recalls' measures under the concept of harmonic mean. The formula of F1-score can be interpreted as a weighted average between precision and recall, where F1-score reaches its best value at $1(100.00)$ and worst score at $0(0.00)$. The relative contribution of precision and recall are equal to the F1-score and the harmonic mean is useful to find the best trade-off between the two quantities ${ }^{53}$.

Thus, for any class $\mathrm{k}$ among the three classes (Extant, Extinct and NPL):

$$
\begin{gathered}
\text { Precision, } \mathrm{P}_{\mathrm{K}}=\frac{T P_{K}}{T P_{K}+F P_{K}} \\
\text { Recall, } \mathrm{R}_{\mathrm{k}}=\frac{T P_{K}}{T P_{K}+F N_{K}} \\
F 1-\text { Score }_{K}=2 * \frac{P_{K} * R_{K}}{P_{K}+R_{K}}
\end{gathered}
$$


The macro-averaged measures of precision, recall and F1-score are the simple average overall classes with equal weight to each incident type while micro average measures are based on the cumulative number of true positives (TP), true negatives (TN), false positives (FP) and false negatives (FN) per studied type ${ }^{54}$.

$$
\begin{gathered}
\text { Macro Precision, } \mathrm{P}_{\text {Macro }}=\frac{\sum_{k=1}^{k} \text { Precision }_{k}}{K} \\
\text { Macro Recall, } \mathrm{R}_{\text {Macro }}=\frac{\sum_{k=1}^{k} \text { Recall }_{k}}{K} \\
F 1-\text { Score }_{\text {Macro }}=2 * \frac{P_{\text {Macro }} * R_{\text {Macro }}}{P_{\text {Macro }}+R_{\text {Macro }}}
\end{gathered}
$$

$$
\begin{gathered}
\text { Micro Precision, } \mathrm{P}_{\text {Micro }}=\frac{T P_{E x t a n t}+T P_{E x t i n c t}+T P_{N P L}}{T P_{E x t a n t}+T P_{E x t i n c t}+T P_{N P L}+F P_{E x t a n t}+F P_{E x t i n c t}+F P_{N P L}} \\
\text { Micro Recall, } \mathrm{R}_{\text {Micro }}=\frac{T P_{E x t a n t}+T P_{E x t i n c t}+T P_{N P L}}{T P_{\text {Extant }}+T P_{\text {Extinct }}+T P_{N P L}+F N_{\text {Extant }}+F N_{E x t i n c t}+F N_{N P L}} \\
F 1-\text { Score }_{\text {Micro }}=2 * \frac{P_{\text {Micro }} * R_{\text {Micro }}}{P_{\text {Micro }}+R_{\text {Micro }}}
\end{gathered}
$$

Class wise performance of the protocol is shown in Fig. 7. Among the three classes, Extant samples were better predicted by the protocol with an F1-score of 97.14. The protocol slightly outperformed in the case of Extinct Class prediction (F1-score: 83.33) than NPL class (F1-score: 85.71). In the proposed protocol, the micro average for recall, precision and F1-score were $89.25,88.38$, and 88.72 respectively. This is a single labelled multi-classification problem therefore in the case of micro average, both the precision and recall are equal to the accuracy of the protocol i.e., $90 \%$ which deduced the harmonic mean i.e., micro F1 score as a similar value (Table 2).

TABLE 2. Performance of MBLDP-R for Different Classes (Class Wise and in Average Measurements).

\begin{tabular}{|c|c|c|c|}
\hline & Recall/Sensitivity & Precision & F1-Score \\
\hline Extant & 94.44 & 100.00 & 97.14 \\
\hline Extinct & 83.33 & 83.33 & 83.33 \\
\hline NPL & 90.00 & 81.82 & 85.71 \\
\hline Protocol $_{\text {Macro }}$ & 89.25 & 88.38 & 88.72 \\
\hline Protocol $_{\text {Micro }}$ & 90.00 & 90.00 & 90.00 \\
\hline
\end{tabular}

The area under the curve (AUC) of receiver operating characteristics (ROC) curve (AUC-ROC) is a performance metric that is based on a varying threshold value. ROC is a probability curve and the area under the curve (AUC) measures separability. In summary, the AUC metric announces the capability of the protocol in distinguishing the classes. AUC ranges from 0 to 1 and higher value of AUC depicts better model ( 1 depicts a perfect model). Mathematically, it can be created by plotting true positive rate (TPR) 
i.e., sensitivity or recall vs false positive rate (FPR) (1- Specificity $\left(\frac{T N}{T N+F P}\right)$ ) on varying threshold values ${ }^{55}$. The AUC metric of the proposed protocol is shown in (Fig. 8). AUC for the curve of Extant class (0.97) is the highest with NPL (0.92) and Extinct (0.88) follows with a little margin among themselves.

Interestingly, the average time required for the completion of the tests was 17.6 minutes while the minimum time was 15.20 minutes and the maximum time was 19.45 minutes. For Extant samples, the time was 17.3 minutes, for Extinct it was 16.8 minutes and for NPL it was 18.2 minutes. Moreover, negative tests took a little longer with an average of 18.4 minutes. Positive protein tests required more time for completion. All three-yes tests took the least time for completion, with an average of 15.2 minutes.

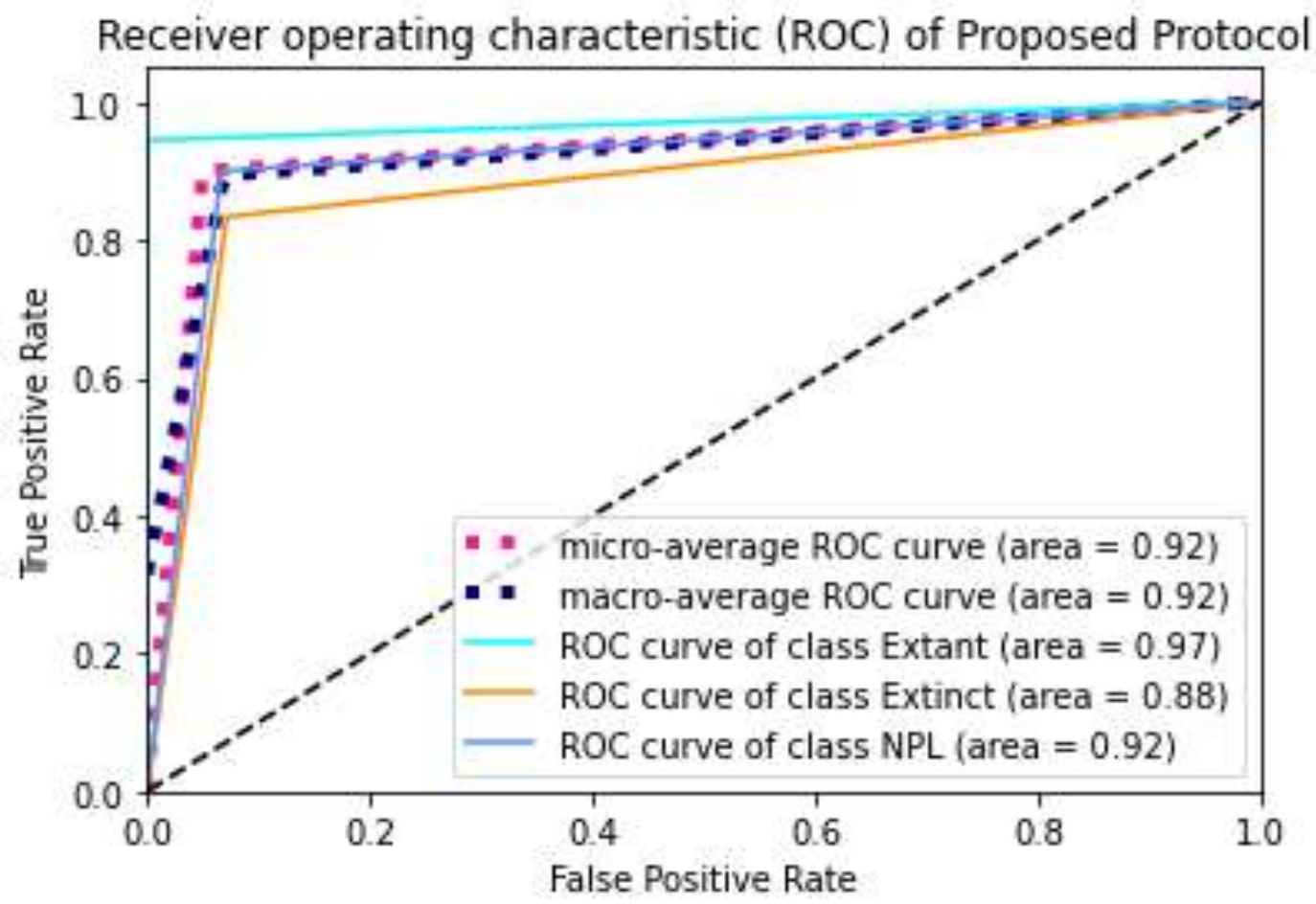

FIG. 8. AUC-ROC of the MBLDP-R.

\section{Discussion and Conclusion}

In compliance with URC 2021 guidelines, a time-efficient, multiple biomolecules (protein, carbohydrate and ammonium ions) based life detection protocol from soil sample analysis (MBLDP-R) has been established through the research work described here. One of the major outcomes of this research is the use of novel validation methodology for multiple biomolecules-based life detection protocols and proposing an improved protocol for classifying the samples with superior performance. According to the available literature, no existing protocol for detecting life in soil samples provides capabilities to this extent. The developed protocol MBLDP-R proposes a multiple biomolecule-based detection method, designed to be embedded as a part of the scientific subsystem of a rover to be used for planetary exploration. A weighted test scoring algorithm has been developed to find the most suitable test method for the given situation. This test scoring method can also be applied in different situations by setting the weights accordingly. To evaluate the proposed protocol, a robust scientific exploration rover subsystem was developed. The evaluation study shows the proposed protocol to be effective in detecting the biosignatures in soil samples and classify them into three desired classes as Extant, Extinct and No Presence of Life (NPL). In contrast to prior works where a single biomolecule-based detection system ${ }^{4-6,11}$ and theoretical framework-based 
contributions $^{7-9}$ were established, the proposed MBLDP-R has shown improvement in many aspects as shown in Table 3. A high recall (89.25), precision (88.38) and F1-Score (88.72) along with an acceptable AUC value (0.92 Micro and 0.97 Macro) from the experimental analysis indicate the effectiveness and reliability of this protocol to detect signatures of life from soil samples. Moreover, an average time of 17.6 minutes for completing a full detection ensured that the requirement of the protocol to be rapid was met.

Unlike the other documented protocols, which are based on a single biomolecule, multiple integrations of biomolecules decrease the possibility of getting false negatives. For example: in the case of the single biomolecule based method of Mora et. al. ${ }^{6}$, a sample would be termed as No Presence of Life (NPL) if an amino acid is absent in that sample. However, other biomolecules, which remain unidentified due to the limitation of the structure of the protocol, cannot contribute to classify the sample. The protocol proposed here is capable of detecting life in the absence of amino acids as it utilizes the presence of both carbohydrates and ammonium ions or only carbohydrates to classify the sample as Extant. A similar statement is also true for the works ${ }^{4,5,11}$ where only nucleic acid has been used to detect the signature of life. The proposed MBLDP-R indicates better performance in classifying samples by considering all possible conditions regarding the presence of proteins, carbohydrates and ammonium ions and generates better conclusions than the previously developed life detection protocols. Additionally, the developed subsystem with the implemented protocol is resource and time efficient unlike some of the prior life detection protocols ${ }^{4,6,11}$. Finally, starting with the development of the protocol, choosing the best tests for biomolecule detection, development of a robotic subsystem capable of sample collection and onboard analysis and lastly, evaluation with the real-time samples demonstrate the holistic approach of this research that can be helpful for other researchers to explore multiple biomolecules-based life detection methods.

TABLE 3. Comparison of MBLDP-R with Earlier Life Detection Protocols.

\begin{tabular}{|c|c|c|c|c|c|c|}
\hline $\begin{array}{l}\text { Developed } \\
\text { Life } \\
\text { Detection } \\
\text { Protocols }\end{array}$ & $\begin{array}{l}\text { Base Theory of } \\
\text { Life Detection }\end{array}$ & $\begin{array}{c}\text { Soil } \\
\text { Sample } \\
\text { Collection } \\
\text { Subsystem }\end{array}$ & $\begin{array}{c}\text { On-Board } \\
\text { Analysis }\end{array}$ & $\begin{array}{c}\text { Time } \\
\text { Efficiency }\end{array}$ & $\begin{array}{c}\text { Evaluation } \\
\text { using } \\
\text { Empirical } \\
\text { Samples }\end{array}$ & $\begin{array}{l}\text { Resource/ } \\
\text { Cost } \\
\text { Efficiency }\end{array}$ \\
\hline $\begin{array}{l}\text { (Mojarro et. } \text { al. 2017) }{ }^{11}\end{array}$ & Nucleic Acid & Yes & Yes & No & Yes & No \\
\hline $\begin{array}{c}(\text { Goordial et } \\
\text { al. 2017) }\end{array}$ & Nucleic Acid & Yes & No & No & Yes & Yes \\
\hline $\begin{array}{l}\text { (Kiflen et al. } \\
2020)^{5}\end{array}$ & Nucleic Acid & Yes & Yes & Yes & No & Yes \\
\hline $\begin{array}{c}\text { (Mora et al. } \\
2020)^{6}\end{array}$ & Amino acid & Yes & Yes & No & Yes & No \\
\hline MBLDP-R & $\begin{array}{c}\text { Multiple } \\
\text { Biomolecule } \\
\text { Based }\end{array}$ & Yes & Yes & Yes & Yes & Yes \\
\hline
\end{tabular}


Even with its efficiency and better performance, the work has some limitations. Firstly, the initial filtration of the biomolecules was based on the guidelines of URC 2021. If there was no time limit, the selected biomolecules could have been different and thereby the principles for test scoring would be different. Secondly, the protocol has been evaluated using a moderately sized sample of 40 . There are future scopes to evaluate this protocol with a large sample size along with an exploration of variations of this protocol. Another limitation is the sensitivity of the protocol. For the detection of the biomolecules, the protocol ensured that the samples to be detected as Extant, Extinct or NPL were exactly that. Therefore, the protocol had no scope to test how sensitive it was in determining the different biomolecules. Whether the protocol would have detected trace amounts of the biomolecules is not apparent in this study, which can be an interesting future scope of this research.

\section{REFERENCE}

1. Speight, J. G. Water systems. in Natural Water Remediation (ed. Speight, J. G.) 1-51 (Elsevier, 2020).

2. Aerts, J. W., Röling, W. F. M., Elsaesser, A. \& Ehrenfreund, P. Biota and biomolecules in extreme environments on Earth: Implications for life detection on Mars. Life 4, 535-565 (2014).

3. Abrahamsson, V. et al. Extraction and separation of chiral amino acids for life detection on Ocean Worlds without using organic solvents or derivatization. Astrobiology 21, 575-586 (2021).

4. Goordial, J. et al. In situ field sequencing and life detection in remote $\left(79^{\circ} 26^{\prime} \mathrm{N}\right)$ Canadian high arctic permafrost ice wedge microbial communities. Front. Microbiol. 8, (2017).

5. Kiflen, M., Shariff, O., Mahdi, H. \& Balsara, F. Novel nucleic acid-based soil sample analysis system for planetary exploration. (2020).

6. Mora, M. F., Kehl, F., da Costa, E., Bramall, N. \& Willis, P. A. Fully automated microchip electrophoresis analyzer for potential life detection missions. Anal. Chem. 92, 12959-12966 (2020).

7. Kite, E. S., Gaidos, E. \& Onstott, T. C. Valuing life-detection missions. Astrobiology 18, 834-840 (2018).

8. Neveu, M., Hays, L. E., Voytek, M. A., New, M. H. \& Schulte, M. D. The Ladder of Life Detection. Astrobiology 18, 1375-1402 (2018).

9. Sharukh, M., Karim, B., Nv, S. \& Thomas, J. Intelligent deployable mini rover from the mars rover for deep narrow scientific investigation. Int. J. Sci. Res. Comput. Sci. Eng. Inf. Technol. 174184 (2019).

10. Rećko, M., Tołstoj-Sienkiewicz, J. \& Turycz, P. Versatile soil sampling system capable of collecting, transporting, storing and preliminary onboard analysis for Mars rover analogue. Solid State Phenom. 260, 59-65 (2017).

11. Mojarro, A., Ruvkun, G., Zuber, M. T. \& Carr, C. E. Nucleic acid extraction from synthetic Mars analog soils for in situ life detection. Astrobiology 17, 747-760 (2017). 
12. Nielsen-Marsh, C. et al. The chemical degradation of bone. in Human Osteology: In Archaeology and Forensic Science (eds. Cox, M. \& Mays, S.) 548 (Cambridge University Press, 2000).

13. Nielsen-Marsh, C. Biomolecules in fossil remains: Multidisciplinary approach to endurance. Biochem. 24, 12-14 (2002).

14. Walworth, J. Nitrogen in soil and the environment. Arizona.edu (2017).

15. Dwek, R. A. Glycobiology: Toward understanding the function of sugars. Chem. Rev. 96, 683-720 (1996).

16. Rudd, P. M. \& Dwek, R. A. Glycosylation: heterogeneity and the $\{3 \mathrm{D}\}$ structure of proteins. Crit. Rev. Biochem. Mol. Biol. 32, 1-100 (1997).

17. Cheshire, M. V. Origins and stability of soil polysaccharide. J. Soil Sci. 28, 1-10 (1977).

18. Debosz, K., Vognsen, L. \& Labouriau, R. Carbohydrates in hot water extracts of soil aggregates as influenced by long-term management. Commun. Soil Sci. Plant Anal. 33, 623-634 (2002).

19. Singh, A. \& Singh, R. \{DNA\}: An important component of life. in Biochemistry, Biophysics, and Molecular Chemistry 195-207 (Apple Academic Press, 2020).

20. Seeman, N. C. \& Sleiman, H. F. \{DNA\} nanotechnology. Nat. Rev. Mater. 3, 17068 (2018).

21. Zhao, Y., Chen, F., Li, Q., Wang, L. \& Fan, C. Isothermal amplification of nucleic acids. Chem. Rev. 115, 12491-12545 (2015).

22. Pääbo, S. Molecular cloning of Ancient Egyptian mummy \{DNA\}. Nature 314, 644-645 (1985).

23. Evershed, R. P. Biomolecular archaeology and lipids. World Archaeol. 25, 74-93 (1993).

24. Cross, B. E., Edinberry, M. N. \& Turner, W. B. Pigments of Gnomonia erythrostoma. I. The structures of erythrostominone, deoxyerythrostominone, and deoxyerythrostominol. J. Chem. Soc. Perkin 1 3, 380-390 (1972).

25. Mizukami, H., Konoshima, M. \& Tabata, M. Variation in pigment production in Lithospermum erythrorhizon callus cultures. Phytochemistry 17, 95-97 (1978).

26. Mumtaz, R., Bashir, S., Numan, M., Shinwari, Z. K. \& Ali, M. Pigments from soil bacteria and their therapeutic properties: A mini review. Curr. Microbiol. 76, 783-790 (2019).

27. Młodzińska, E. Survey of plant pigments: Molecular and environmental determinants of plant colors. Abcbot.pl (2009).

28. de Marsac, N. Phycobiliproteins and phycobilisomes: the early observations. Photosynth. Res. 76, 193-205 (2003).

29. Jones, J. G. Studies on lipids of soil micro-organisms with particular reference to hydrocarbons. $J$. Gen. Microbiol. 59, 145-152 (1969).

30. Stevenson, F. J. Lipids in soil. J. Am. Oil Chem. Soc. 43, 203-210 (1966). 
31. URC2020 Science Discussion. Youtube https://www.youtube.com/watch?v=jW21VtoHCag (2020).

32. Serra, J. A. Histochemical tests for proteins and amino acids; the characterization of basic proteins. Stain Technol. 21, 5-18 (1946).

33. Lubran, M. M. The measurement of total serum proteins by the Biuret method. Ann. Clin. Lab. Sci. 8, 106-110 (1978).

34. Domon, B. \& Aebersold, R. Mass spectrometry and protein analysis. Science (80-. ). 312, 212-217 (2006).

35. Potter, R. S. \& Snyder, R. S. The determination of ammonia in soils. J. Ind. Eng. Chem. 7, 221226 (1915).

36. Kwak, D., Lei, Y. \& Maric, R. Ammonia gas sensors: A comprehensive review. Talanta 204, 713-730 (2019).

37. Katoch, R. Carbohydrate Estimations. in Analytical Techniques in Biochemistry and Molecular Biology 67-76 (Springer New York, 2011).

38. Bakken, L. R. \& Frostegård, Å. Nucleic acid extraction from soil. in Soil Biology 49-73 (Springer Berlin Heidelberg, 2006).

39. Jensen, A. et al. Quantitative Paper Chromatography of Carotenoids. Acta Chem. Scand. 13, 1863-1868 (1959).

40. Strain, H. H., Sherma, J., Benton, F. L. \& Katz, J. J. One-way paper chromatography of the chloroplast pigments of leaves. Biochim. biophys. acta 109, 1-15 (1965).

41. Brotas, V. \& Plante-Cuny, M.-R. The use of $\{$ HPLC $\}$ pigment analysis to study microphytobenthos communities. Acta Oecol. 24, S109--S115 (2003).

42. Suzuki, Y. \& Shioi, Y. Identification of chlorophylls and carotenoids in major teas by highperformance liquid chromatography with photodiode array detection. J. Agric. Food Chem. 51, 5307-5314 (2003).

43. Yuan, J., Zhang, Y., Shi, X., Gong, X. \& Chen, F. Simultaneous determination of carotenoids and chlorophylls in algae by high performance liquid chromatography. Se Pu 15, 133-135 (1997).

44. Portable high performance liquid chromatography. Axcendcorp.com (2020).

45. Mubarak, M., Shaija, A. \& Suchithra, T. V. A review on the extraction of lipid from microalgae for biodiesel production. Algal Res. 7, 117-123 (2015).

46. Patel, A. et al. Lipids detection and quantification in oleaginous microorganisms: an overview of the current state of the art. BMC Chem Eng 1, (2019).

47. Oades, J. M. Soil organic matter and structural stability: mechanisms and implications for management. Plant Soil 76, 319-337 (1984).

48. F. M. Swain (2), M. A. Rogers (3),. Distribution of carbohydrate residues in some fossil specimens 
and associated sedimentary matrix and other geologic samples. J. Sediment. Res. 37, 12-24 (1967).

49. Palacas, J. G., Swain, F. M. \& Smith, F. Presence of carbohydrates and other organic compounds in ancient sedimentary rocks. Nature 185, 234 (1960).

50. Briggs, D. E. G. \& Summons, R. E. Ancient biomolecules: their origins, fossilization, and role in revealing the history of life: Prospects \\& Overviews. Bioessays 36, 482-490 (2014).

51. Capone, D. G., Popa, R., Flood, B. \& Nealson, K. H. Geochemistry. Follow the nitrogen. Science (80-. ). 312, 708-709 (2006).

52. MIST Mongol Barota - URC SAR 2021. Youtube https://www.youtube.com/watch?v=0ezyJEdleQM (2021).

53. Grandini, M., Bagli, E. \& Visani, G. Metrics for multi-class classification: An overview. (2020).

54. Sokolova, M. \& Lapalme, G. A systematic analysis of performance measures for classification tasks. Inf. Process. Manag. 45, 427-437 (2009).

55. Narkhede, S. Understanding auc-roc curve. Towar. Data Sci. 26, 220-227 (2018).

\section{Acknowledgements}

The Authors are extremely grateful to the Department of Computer Science and Engineering (CSE), Department of Mechanical Engineering and Faculty of Science \& Engineering, Military Institute of Science and Technology, Dhaka, Bangladesh for laboratory and administrative support along with constant coordination throughout the system development. Moreover, a heartfelt thanks to The Mars Society for hosting such a well-arranged competition, University Rover Challenge 2021 and providing this young team of researchers a chance to prove their potentiality and research aptitude.

\section{Author contributions}

AZ: Methodology, Evaluation Study, Writing - Original Draft; FA: Investigation, Software, Writing - Original Draft; HK: Supervision, Writing- Original Draft, Experiment Design FNA: Resources, Investigation, Writing; OS: Design, Fabrication, Writing; AMR: Design, Fabrication; SNN: Investigation, Validation, Writing; HMC: Investigation, Validation;

MR: Supervision, Writing- Reviewing and Editing;

\section{Data availability statement}

The data that support the findings of this study are available from the corresponding author, [MR], upon reasonable request. 


\section{Additional Information}

\section{Author Disclosure Statements}

The authors declare that they have no known competing financial interests, personal relationship, or organizational relationships or employment related interests that inappropriately affect the integrity of the research reported in this paper.

\section{Funding Statement}

No funding to report for this submission 